\title{
The phenotypic variability of amyotrophic lateral sclerosis
}

\author{
Bart Swinnen and Wim Robberecht
}

\begin{abstract}
Classic textbook neurology teaches that amyotrophic lateral sclerosis (ALS) is a degenerative disease that selectively affects upper and lower motor neurons and is fatal 3-5 years after onset-a description which suggests that the clinical presentation of ALS is very homogenous. However, clinical and postmortem observations, as well as genetic studies, demonstrate that there is considerable variability in the phenotypic expression of ALS. Here, we review the phenotypic variability of ALS and how it is reflected in familial and sporadic ALS, in the degree of upper and lower motor neuron involvement, in motor and extramotor involvement, and in the spectrum of ALS and frontotemporal dementia. Furthermore, we discuss some unusual clinical characteristics regarding presentation, age at onset and disease progression. Finally, we address the importance of this variability for understanding the pathogenesis of ALS and for the development of therapeutic strategies.
\end{abstract}

Swinnen, B. \& Robberecht, W. Nat. Rev. Neurol. advance online publication 14 October 2014; doi:10.1038/nrneurol.2014.184

\section{Introduction}

Amyotrophic lateral sclerosis (ALS) is a heterogeneous disorder. The genetic heterogeneity is obvious, given the long list of genes in which mutations cause ALS. ${ }^{1}$ Considering that the aetiology of ALS remains unknown in nearly $90 \%$ of cases, the true causal heterogeneity is likely to be even larger. The mechanistic heterogeneity of ALS adds to the complexity of this disorder. ${ }^{2}$ The fact that ALS starts in the bulbar region in some patients, and in the limbs in others, has always been evident to clinicians, and is often used as stratification parameter in clinical trials. Phenotypic variability goes far beyond the site of onset, however, and can be observed with regard to age at onset, familial occurrence, type of motor neuron involvement, extent of extramotor involvement, and disease duration, among other parameters. Here, we will review several aspects of this phenotypic heterogeneity of ALS and discuss some of its implications.

\section{A student's vignette: classic ALS Variability in location of onset \\ Spinal onset}

In most patients, ALS starts around the age of 60 years with asymmetric, painless weakness in a limb, referred to as spinal-onset ALS (Figure 1). ${ }^{3,4}$ Clinical examination usually reveals atrophy and weakness of muscles, fasciculations, hyperreflexia (or at least brisk reflexes), and often a mild to severe hypertonia. ${ }^{3}$ Clinicians designate weakness, muscle atrophy and fasciculations as lower motor neuron signs, whereas hyperreflexia and hypertonia indicate upper motor neuron involvement (Figure 2). Interestingly, the Babinski sign-also known as the extensor plantar reflex-is often absent, and

\section{Competing interests}

The authors declare no competing interests. hypertonia is often 'glue-like', reminiscent of what one finds in frontal or even extrapyramidal syndromes, and different from the clasp-knife phenomenon of the upper limbs in stroke and the spastic kick of the lower limbs in familial spastic paraparesis. Initially, the abnormal findings can be patchy, but the disorder spreads relentlessly over time. In many but not all patients, this spread seems contiguous, a phenomenon hypothesized by some authors to reflect the spread at the cellular level. ${ }^{5}$

\section{Bulbar onset}

In about $20 \%$ of patients with ALS, the weakness starts in bulbar muscles, with dysarthria, dysphagia and tongue fasciculations (Figure 2) ${ }^{3,6} \mathrm{~A}$ brisk jaw jerk is often found in these patients, and pseudobulbar affect (an inaccurate term mainly referring to uncontrolled crying or laughing) is sometimes present. The presence of limb hyperreflexia suggests that the disease has already spread. Bulbar-onset ALS was originally considered to be a different disease from ALS, and was termed 'progressive bulbar palsy.?

The onset of symptoms is gradual in most patients, but some become aware of their problems quite abruptly; neurologists often need to consider stroke and myasthenia in the differential diagnosis of these patients. Bulbaronset ALS must also be distinguished from Kennedy's disease (bulbar and spinal muscular atrophy) or from Brown-Vialetto-Van Laere syndrome (see below). ${ }^{3}$

Patients with bulbar-onset ALS have a worse prognosis than patients with spinal onset, with a mean survival of 2 years and long-term (>10 years) survival of only $3 \%{ }^{6}$ The poor prognosis is mostly attributable to the fact that patients with bulbar-onset ALS are prone to aspiration and nutritional problems, and is possibly also related to earlier respiratory dysfunction due to involvement of the cervical 


\section{Key points}

- Amyotrophic lateral sclerosis (ALS) is a highly heterogeneous entity

- Cognitive impairment is a common feature of ALS: frontotemporal dementia and ALS constitute the ends of a spectrum reflecting different manifestations of the same pathogenic mechanism

- Upper and lower motor neuron involvement is variable in ALS, and yields a spectrum with primary lateral sclerosis and progressive muscular atrophy at the two ends

- In rare cases, extrapyramidal, cerebellar, sensory and autonomic systems can be affected in ALS, indicating that ALS should be seen as a multisystem neurodegenerative disease

- The method and timing of assessment of a patient account for a considerable proportion of the clinical variability

- The biology underlying the ALS phenome needs to be elucidated, as the pathophysiological mechanisms of the disease could be targets for therapeutic interventions a

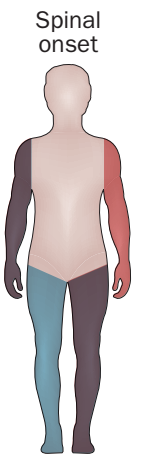

e Pseudopolyneuritic

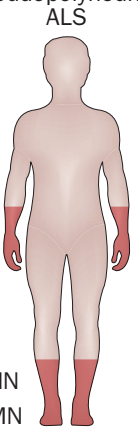

\section{b}

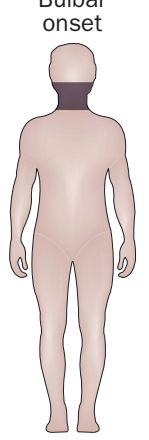

Hemiplegic

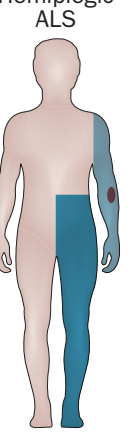

c Progressive muscular atrophy

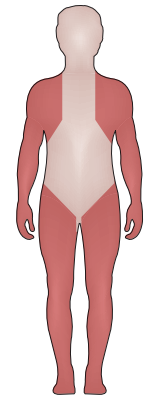

g

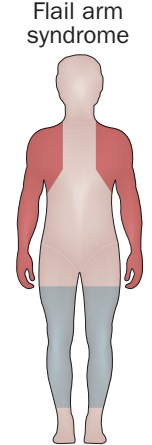

d Primary lateral sclerosis

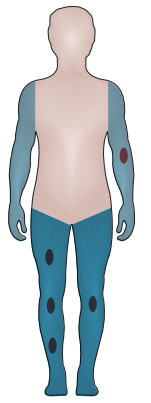

h Flail leg syndrome

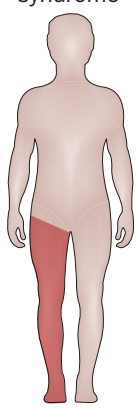

Figure 1 | Pattern of motor involvement in different ALS phenotypes. Red indicates LMN involvement, blue indicates UMN involvement. Darker shading indicates moresevere involvement. a | In spinal-onset ALS, patchy UMN and LMN involvement is observed in all limbs. b | In bulbar-onset ALS, UMN and LMN involvement is observed in the bulbar muscles. c | In progressive muscular atrophy, LMNs in arms and legs are involved, often proximally. d | In primary lateral sclerosis, UMNs of arms and legs are primarily involved, but later in the disease, discrete LMN involvement can be detected. e | In pseudopolyneuritic ALS, only LMNs restricted to the distal limbs are involved. f $\mid$ In hemiplegic ALS, unilateral UMN involvement with sparing of the face, and sometimes discrete LMN involvement, can be observed. $\mathbf{g}$ | In flail arm syndrome, LMN involvement is restricted to the upper limbs, but mild UMN signs can be detected in the legs. h | In flail leg syndrome, $\mathrm{LMN}$ involvement is restricted to the lower limbs, and is often asymmetric.

Abbreviations: ALS, amyotrophic lateral sclerosis; LMN, lower motor neuron; UMN, upper motor neuron.

\section{Respiratory onset}

In about $3-5 \%$ of patients, ALS is characterized by respiratory onset, with orthopnoea or dyspnoea, and mild or even absent spinal or bulbar signs. ${ }^{6}$ Respiratoryonset ALS has a male predominance. ${ }^{6,10}$ The prognosis is notoriously poor, with a mean survival of 1.4 years and no long-term survival., ${ }^{6,10}$

\section{Genes and type of onset}

The factors that determine the nature of ALS onset remain unknown. Sex hormones are suspected to contribute to the type of onset because of the striking female predominance in bulbar-onset patients. ${ }^{9}$ Bulbar onset is very frequent in ALS associated with C9orf72 mutations; by contrast, ALS associated with mutations in superoxide dismutase 1 (SOD1) almost never starts in the bulbar motor neurons (see below). Such associations between the type of onset and genetic causes suggest that the site of onset is unlikely to merely reflect a stochastic process.

\section{Familial versus sporadic ALS}

About $10 \%$ of patients with ALS have an affected relative and are, thus, considered to have familial ALS. ${ }^{1}$ Those who do not have an affected relative are considered to have sporadic ALS. It is likely that many if not all cases of familial ALS will turn out to be hereditary in nature, because in a growing number of the pedigrees of patients with familial ALS, a causal mutation is eventually identified. In some populations, such a hereditary cause is identifiable in nearly $80 \%$ of familial ALS cases. ${ }^{1}$ Mutations in SOD1, TARDBP (the gene that encodes TAR DNA-binding protein 43 [TDP-43]), FUS and C9orf72 explain more than half of cases of familial ALS. ${ }^{1}$

The cause of sporadic ALS is unknown, but twin studies suggest that it has a genetic component as well, possibly in interaction with as yet unknown environmental factors. ${ }^{11}$ Some patients with sporadic ALS initially receive a wrong classification, as they are later found to have a hereditary form of the disease when they are found to carry a causal mutation. Reduced penetrance and a low number of offspring are the main explanations for the misclassifications, ${ }^{13}$ but other contributory factors include a lack of complete information of the medical history of the patients' relatives, non-paternity events, and variability of the phenotypic expression. ${ }^{14}$ In most populations, nearly $10 \%$ of the patients with so-called sporadic ALS carry a C9orf72 mutation; ${ }^{1}$ the advanced age at which C9orf72-associated ALS often begins and unexpected variability of the phenotype associated with C9orf72 mutations (see below) are the main reasons why these patients are misclassified.

\section{Age at onset}

Although ALS usually starts in the fifth or sixth decade of life, onset at almost any age has been described. Juvenile ALS is defined as ALS with age at onset before 25 years, and the course of progression is generally slower than in other forms of ALS. ${ }^{15,16}$ Mutations in ALS2, SETX and FUS are well-known causes of juvenile ALS.,14 Patients carrying the FUS Pro525Leu mutation have a notoriously phrenic motor neurons. ${ }^{8}$ Loss of ambulation is an ominous sign for these patients, indicating mean life expectancy of only 3 months. ${ }^{9}$ The site of onset is, therefore, used as a stratification parameter in clinical trials. 


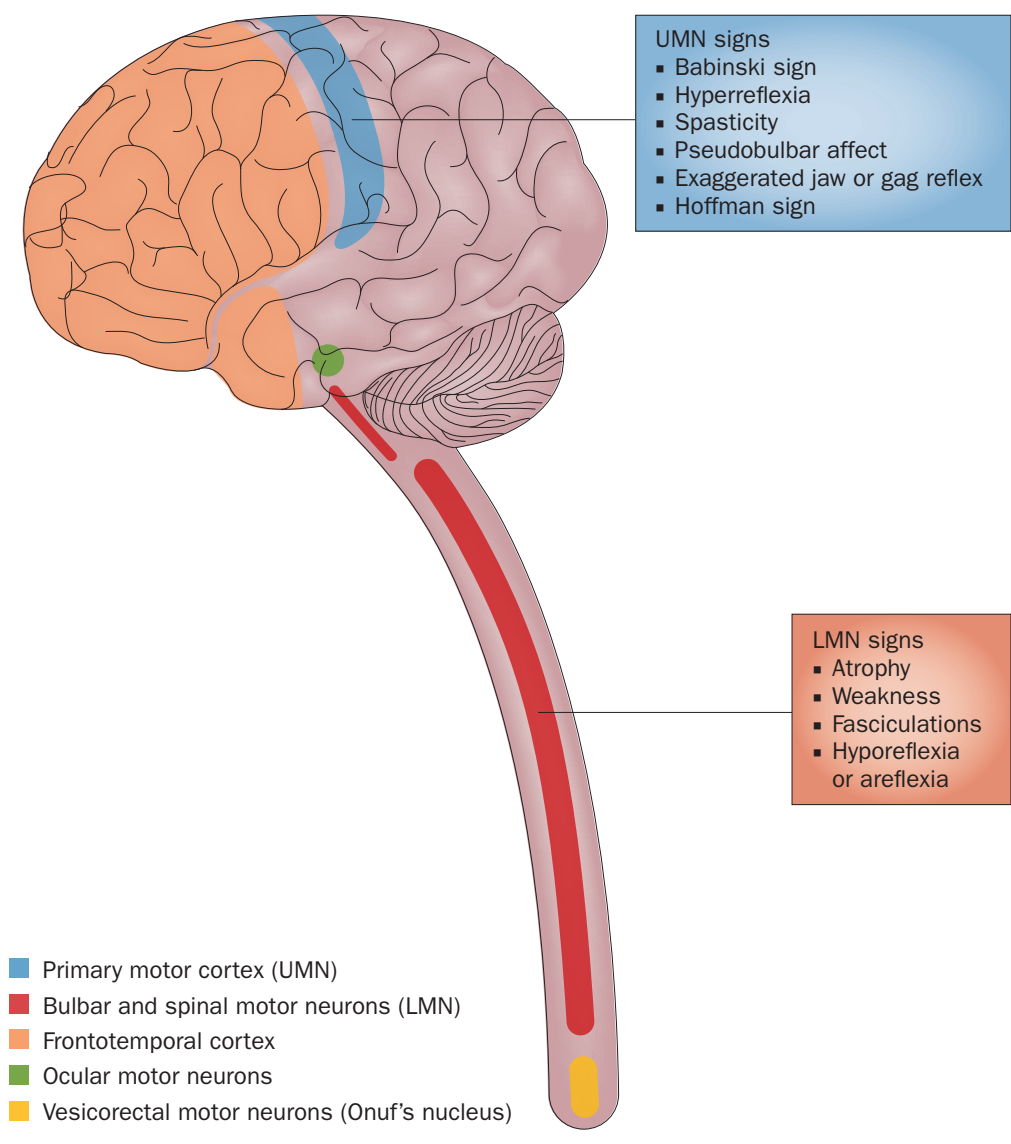

Figure 2 | Preferential sites of neuronal involvement in ALS. UMNs in the primary motor cortex (blue) and bulbospinal LMNs (red) are the preferentially affected sites in ALS. Neurons in the frontotemporal cortex (orange), however, are frequently involved as well; the resulting phenotype will be in the frontotemporal dementia-ALS spectrum. Ocular (green) and vesicorectal (yellow) motor neuron involvement is rare, and happens mostly in cases of longstanding disease. Abbreviations: ALS, amyotrophic lateral sclerosis; LMN, lower motor neuron; UMN, upper motor neuron.

poor prognosis. ${ }^{17-19}$ In some forms of ALS, such as the SETX-associated ALS type 4, the phenotype is so atypical that some authors suggest they should be considered as separate disease entities rather than ALS. ${ }^{20,21}$

$60 \%$ of patients with disease onset between 20 and 40 years of age have predominantly upper motor neuron involvement, and relatively few of these patients (15\%) have bulbar-onset disease. ${ }^{15}$ Older age at onset is associated with decreased likelihood of upper motor neuron involvement (20\%), increased probability of bulbar onset (some studies report up to $50 \%$ with onset after 80 years of age), and poor prognosis. ${ }^{9,22,23}$ Onset after 80 years is associated with a particularly short survival. ${ }^{16}$

\section{Rate of progression}

Although median survival in ALS is generally around 3 years from diagnosis, variability in survival is remarkable and reflects the variability in the rate of disease progression. The difference in individual rates of functional decline, even in small series of patients, demonstrates this high variability (Figure 3), and is one of the factors that complicate clinical trials in ALS.
At one end of this continuum are the $10 \%$ of patients who live longer than 10 years with ALS. ${ }^{16}$ At autopsy, their disease does not differ from classic ALS. ${ }^{24}$ Long survival is seen more frequently in patients with juvenile ALS and upper motor neuron-predominant ALS. ${ }^{16,23}$ Some forms of hereditary ALS are known to be associated with particularly long or short survival. For example, long survival has been reported in association with the SOD1 Asp90Ala mutation, whereas the SOD1 Ala4Val mutation can induce a very aggressive disease. ${ }^{1}$ Patients with a hexanucleotide (GGGGCC) expansion mutation in the C9orf72 gene have shorter survival than the average ALS patient. ${ }^{25-28}$

Even within families in which members carry the same mutation, variability in progression rates is large. ${ }^{29-31}$ This finding suggests that there is no straightforward relationship between the genetic cause and phenotype but, rather, that factors-either genetic or environmental-modify the phenotypic expression, and in particular age at onset and disease progression. It is important to identify these modifying factors, as they could be targets for therapeutic intervention, even in the absence of knowledge of the cause of disease. Delaying age at onset or attenuating progression rate and, thus, functional decline is of obvious therapeutic interest. Several small animal models, such as Drosophila and zebrafish, have been used to screen for such modifiers, resulting in the identification of 'druggable' targets or even novel causes of ALS.

\section{Motor neuron involvement Lower motor neuron dominance}

Evidence of both upper and lower motor neuron involvement is required for the diagnosis of ALS, and has been incorporated in the so-called El Escorial criteria. ${ }^{32}$ Of note, however, these criteria were developed to reduce the phenotypic variability in clinical trials, and were not intended for clinical decision-making.

Assessing the involvement of upper motor neurons relies on clinical judgement-largely, evaluation of the briskness of deep tendon reflexes. Neurologists sometimes disagree on the interpretation of this measure: some consider a preserved reflex in an otherwise atrophic muscle to be a sign of upper motor neuron involvement, while others require the reflex to be hyperactive to reach the same conclusion. A patient can, thus, be considered to have ALS by one clinician, and to have progressive muscular atrophy (PMA) by another (Figures 2 and 4).

\section{Progressive muscular atrophy}

PMA refers to an adult-onset lower motor neuron disorder that differs from the rare instances of adult-onset spinal muscular atrophy (SMA) in several respects: PMA is usually asymmetric, can have distal and/or proximal onset, and progresses much faster than SMA. ${ }^{3}$ Thus, PMA essentially equals ALS minus (convincing) upper motor neuron findings. PMA is believed to constitute about $5 \%$ of all patients with motor neuron disease (MND). ${ }^{33}$ The question arises as to whether PMA and ALS have different pathophysiological backgrounds and thereby represent different disorders, or whether PMA represents the end of a spectrum of lower versus 


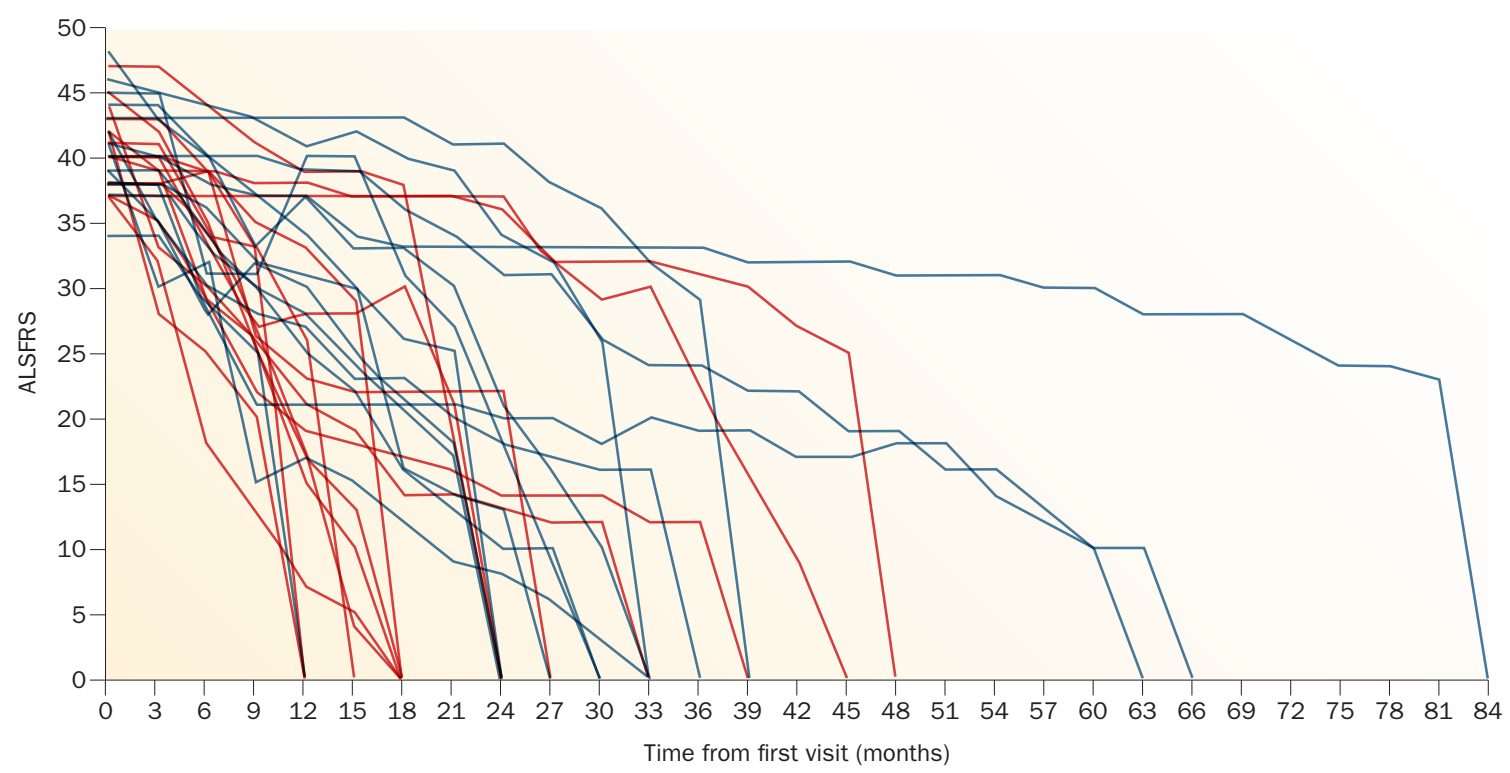

Figure 3 | Variability of disease progression in ALS. ALSFRS scores of 30 randomly selected patients (Leuven University Hospital, Belgium) with ALS, from first visit to death. Patients with bulbar-onset disease are indicated in red, patients with spinal-onset disease are indicated in blue. Abbreviations: ALS, amyotrophic lateral sclerosis; ALSFRS, ALS Functional Rating Scale.

upper motor neuron involvement (Figure 4). Support for the latter hypothesis comes from the finding that some SOD1 mutations give rise to a syndrome that primarily affects lower motor neurons: patients with the SOD1 Ala4Val mutation are classified as having ALS, but they lack upper motor neuron involvement. ${ }^{34,35}$ Furthermore, imaging studies have shown widespread, progressive frontal abnormalities in PMA that are similar to those in ALS, ${ }^{36,37}$ and postmortem studies of patients with lower motor neuron signs only often show classic lateral spinal cord involvement. ${ }^{38}$ Patients with PMA fare slightly better than those with clinical evidence of both lower and upper motor neuron involvement (see below).

\section{Flail arm syndrome}

Some patients have lower motor neuron involvement that remains limited to the upper limbs for at least 12 months (Figure 2). These limbs are often non-functional, while the lower limbs remain normal, although hyperreflexia and some hypertonia may be present. ${ }^{39,40}$ This variant is called flail arm syndrome (also referred to as the scapulohumeral form of ALS, Vulpian-Bernart syndrome, hanging arm syndrome, neurogenic man-in-a-barrel syndrome or brachial amyotrophic diplegia), and has a striking male predominance, with a male:female ratio of $4: 1 .^{40,41}$ After about 20 months, almost all patients with this syndrome develop more-widespread disease; the prognosis is somewhat better than that of classic ALS, with mean survival of 4 years and long-term survival of $17 \%{ }^{6}$

\section{Flail leg syndrome and dropped head syndrome}

Even less frequent than flail arm syndrome is flail leg syndrome, characterized by often asymmetric, and primarily distal, lower motor neuron involvement in the lower limbs for at least 12 months. ${ }^{41}$ Subtle upper motor neuron signs usually emerge over time, ${ }^{41,42}$ and after a mean of 16 months, the upper limbs and bulbar region also become affected. ${ }^{6}$ Progression is slightly slower than in classic ALS. ${ }^{6,41,42}$ Flail leg syndrome with mainly distal and bilateral involvement is also referred to as the 'pseudopolyneuritic', 'Marie-Patrikios' or 'peroneal' form of ALS. ${ }^{41,42}$

Equally rare is onset in the cervical region, limited to the extensors of the neck, resulting in dropped head syndrome. ${ }^{43}$ Such clinical presentation needs to be differentiated from myasthenia gravis or a (typically inflammatory) myopathy.

\section{Upper motor neuron dominance}

Some patients present with upper motor neuron findings only and, therefore, do not meet the standard criteria for ALS. These patients are considered to have primary lateral sclerosis (PLS); however, most of these individuals gradually develop lower motor neuron involvement. ${ }^{44,45}$ The diagnosis of PLS can, therefore, only be made after a sufficiently long period of observation, as $23 \%$ of those who eventually develop lower motor neuron signs do so only after 4 years. ${ }^{44}$

PLS constitutes about $5 \%$ of all cases of MND. ${ }^{46}$ This condition is characterized by slower progression, moreprolonged retention of functionality, sparing of respiratory function and less-severe weight loss than seen in ALS. ${ }^{47}$ Even in patients with PLS, lower motor neuron involvement is often evident at autopsy, ${ }^{46}$ which, together with the fact that known ALS-causing mutations can present with upper motor neuron involvement only, ${ }^{1}$ suggests that PLS could be considered to be at one end of a spectrum of upper motor neuron involvement, with PMA being at the other end (Figure 4).

Interestingly, upper motor neuron involvement can be strikingly asymmetric. An extreme form is the unusual hemiplegic variant also called Mills syndrome or 
a

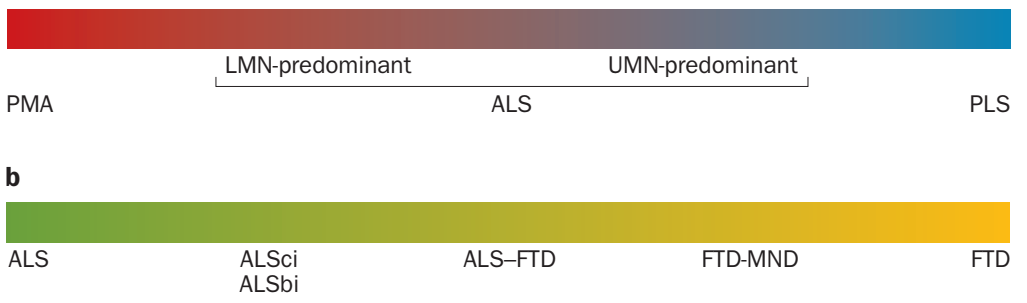

c

Classic ALS

ALS with multisystem degeneration

Extramotor involvement

Figure 4 | ALS, a spectrum disorder. a | PMA (isolated LMN involvement) and PLS (isolated UMN involvement) constitute the ends of a spectrum of LMN and UMN involvement; intermediate phenotypes are considered to be different expressions of ALS. $\mathbf{b} \mid$ ALS and FTD constitute the ends of a spectrum of motor neuron and frontotemporal neuron involvement. This spectrum includes patients with ALS who express isolated ALSci or ALSbi, and patients with ALS who meet the Neary criteria for FTD and are, thus, diagnosed with ALS-FTD. Some patients with FTD have insufficient motor neuron involvement for a diagnosis of ALS, and are classified as FTD-MND. c | A spectrum of extramotor involvement is possible in ALS, ranging from classic ALS with no to mild extramotor involvement to ALS with extrapyramidal, cerebellar, sensory, autonomic, urinary or oculomotor involvement, designated as 'ALS with multisystem degeneration'. Abbreviations: ALS, amyotrophic lateral sclerosis; ALSbi, ALS with behavioural impairment; ALSci, ALS with cognitive impairment; FTD, frontemporal dementia; LMN, lower motor neuron; MND, motor neuron disease; PLS, primary lateral sclerosis; PMA, progressive muscular atrophy; UMN, upper motor neuron.

progressive hemiplegia. ${ }^{48,49}$ This form usually begins with unilateral upper motor neuron involvement in the lower limb, followed by slowly progressive ipsilateral involvement of the arm, with relative sparing of the face. After a variable time period, the disease spreads to the initially unaffected side.

To conclude, several lines of evidence suggest that ALS, PMA and PLS are expressions of one biological entity: patients in the same family, or patients carrying mutations in the same ALS-associated allele, can have classic ALS, PMA or PLS; the clinical characteristics of a patient can vary over time; and detection of upper and lower motor neuron involvement depends on the method of assessment. The main reason for distinguishing these phenotypes is the difference in prognosis. Patients with isolated lower motor neuron involvement live only slightly longer than patients with clinical evidence of both upper and lower motor neuron involvement, ${ }^{50,51}$ whereas patients with PLS, on average, live longer than those with ALS. ${ }^{47}$ The mean survival of ALS patients with predominant upper motor neuron signs -6 years, with about $30 \%$ of cases exhibiting long-term ( $>10$ years) survival-lies between that of PLS and classic ALS. ${ }^{6,44}$ This difference may simply reflect the biological burden of disease (Figure 5). It also implies that the best approach is to study patients with both lower and upper motor neuron involvement only if one intends to (prognostically) homogenize a study population, as is needed, for example, in clinical trials that investigate the effects of a drug on function or survival.

\section{Nonmotor involvement in ALS}

ALS has long been believed to be a neurodegenerative disorder with isolated motor neuron involvement. Absence of cognitive deterioration was initially even postulated as a prerequisite for a diagnosis of ALS. Nowadays, however, it is evident that other neurological systems can be affected, with cognitive impairment being the most frequent manifestation of nonmotor involvement.

\section{Cognitive impairment}

Over the past few decades, a spectrum of cognitive involvement has been increasingly acknowledged as a part of ALS. ${ }^{52-54}$ Degenerative changes in layer V of the frontotemporal lobe represent the anatomical substrate of this cognitive decline, hence the name frontotemporal lobar degeneration (FTLD). Up to $25 \%$ of patients with ALS meet all criteria for the clinical manifestation of FTLD, frontotemporal dementia (FTD), mostly of the behavioural variant. ${ }^{55,56}$ Mild to moderate frontal dysfunction or language abnormalities are even more frequent, but can escape routine clinical examination and be detected on detailed testing only. Apathy (seen in $60 \%$ of patients), disinhibition, delusions and stereotypic behaviour are the most frequently encountered behavioural changes. ${ }^{52,55}$ Whether depression (reported in up to $75 \%$ of patients ${ }^{57}$ ) can also be attributed to the ALS pathology is difficult to judge, as patients have obvious reasons for reactive depression. It should be kept in mind that even mild frontal impairment can contribute to non-compliance with percutaneous endoscopic gastrostomy therapy and noninvasive ventilation, difficulties handling communication machines, psychosocial problems and decision-making, and undoubtedly increases caregiver burden..$^{52,53}$

\section{ALS-FTD spectrum}

ALS is now accepted to constitute a continuum with FTD. Pure ALS (without any evident cognitive abnormality) and pure FTD (without any obvious motor abnormality) are located at the opposite ends of this spectrum (Figure 4). ALS patients with mild behavioural dysfunction are classified as having ALS with behavioural impairment (ALSbi), ${ }^{52}$ whereas patients with mild executive and language dysfunction are said to have ALS with cognitive impairment (ALSci). ${ }^{52}$ Patients with ALS who meet the Neary criteria for FTD are considered to have ALS-FTD. Of note, up to $50 \%$ of patients with a diagnosis of FTD have some motor neuron involvement (which may go unnoticed by the patient), and are said to have 'FTD-MND', 52,54

In ALS, the onset of cognitive problems usually precedes that of motor dysfunction. ${ }^{53,54}$ Frontotemporal involvement can only become evident in retrospect, when motor problems bring the patient under medical attention. Interestingly, eye movement disorders, probably related to frontal network dysfunction, are especially prevalent in these patients. ${ }^{52}$

The prognosis of patients with subtle cognitive impairment is similar to that in classic ALS, ${ }^{55}$ but patients with ALSbi or ALS-FTD have a worse prognosis than do patients with ALS alone. ${ }^{53}$ The life expectancy in ALSFTD is 2.4 years from disease onset, approximately 1 year 


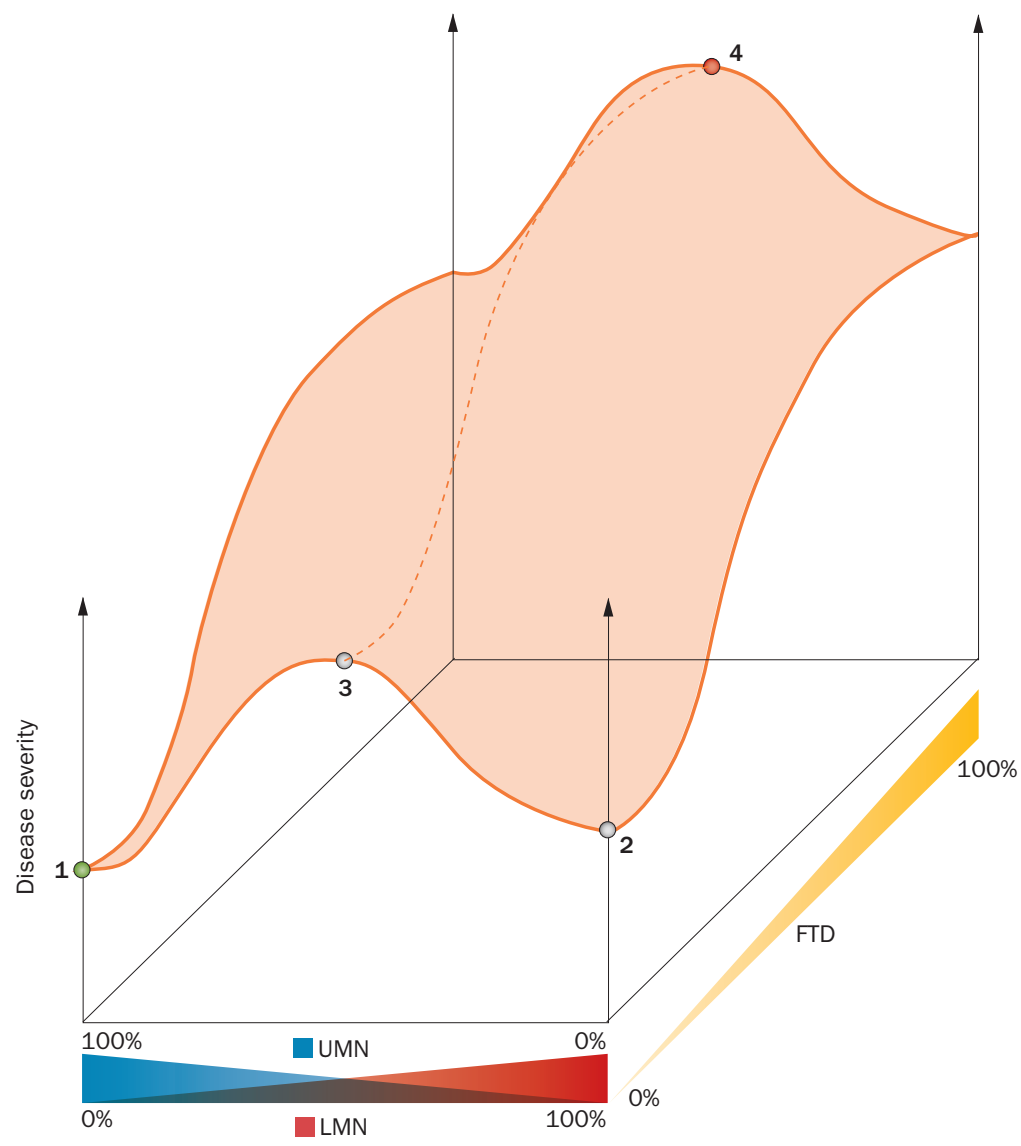

Figure $\mathbf{5}$ | Disease severity correlates with extent of neuronal involvement. In ALS, UMN, LMN and frontotemporal neurons are affected to variable extents, resulting in a wide range of clinical representations and disease severity, which is reflected by large prognostic variability. The horizontal axis depicts the proportion of UMN versus LMN involvement, and frontotemporal involvement is depicted in the diagonal axis. Disease severity is depicted in the vertical axis, and correlates inversely with survival. In the UMN-LMN spectrum, pure UMN involvement, as in PLS (1), and pure LMN involvement, as in PMA (2) have a better prognosis than disease with both UMN and LMN involvement (3). Increasing frontotemporal involvement is associated with shortened survival. Degeneration of all three neuronal systems (4) is associated with the highest disease severity and, hence, the worst prognosis. Abbreviations: ALS, amyotrophic lateral sclerosis; FTD, frontotemporal dementia; LMN, lower motor neuron; PLS, primary lateral sclerosis; PMA, primary muscular atrophy; UMN, upper motor neuron.

less than in pure ALS. ${ }^{58}$ Problems with compliance with therapy and support could contribute to this difference. ${ }^{52,59}$ Alternatively, the difference in prognosis might simply reflect the disease burden (Figure 4).

\section{Lessons from C9orf72 expansions}

The clinical observations discussed above establish a clear link between ALS and FTD. Pathogenic evidence for such link came from the observation that very similar aggregates containing TDP-43, among other proteins, can be found in brain tissue in the majority of patients with either FTD or ALS. ${ }^{60}$ However, it was the discovery of a hexanucleotide expansion mutation in the C9orf72

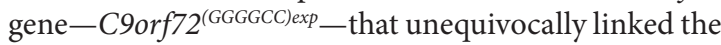
two ends of the spectrum. ${ }^{61-63}$ This mutation underlies nearly half of cases of familial ALS and a quarter of cases of familial FTD. ${ }^{1}$ The pathogenic mechanisms underpinning the connection between $C 90 r f 72^{\text {(GGGGCC)exp }}$ and ALS-FTD remain poorly understood, but the studies connecting the two have already changed our perceptions of these neurodegenerative disorders. ${ }^{2}$

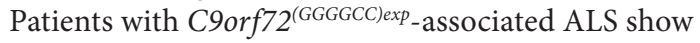
marked phenotypic variability. In patients from within the same family with a C9orf72 expansion, onset can be frontotemporal, bulbar or spinal. ${ }^{64,65}$ The length of the repeat section does not seem to correlate with the severity of disease or site of onset, and patients who are homozygous for the mutation do not have more-severe disease than heterozygous patients. ${ }^{64}$ Bulbar onset is more frequent in ALS patients with C9orf72(GGGGCC)exp than in those without. ${ }^{64}$ Interestingly, bulbar onset is (almost) never seen in mutant-SOD1-associated ALS, and cognitive impairment is equally rare in patients with this mutation. ${ }^{12,66,67}$ It should be noted that cognitive assessment in patients with bulbar failure can be challenging because of the speech problems and pseudobulbar affect that these patients experience, and instruments for bedside evaluation are still being developed.

The spectrum of neuropsychiatric symptoms associated with C9orf72(GGGGCC)exp-associated ALS is much broader than previously thought, even encompassing psychosis and depressive pseudodementia. ${ }^{53,64,65,68,69}$ Furthermore, motor symptoms in these patients can be variable, manifesting as ataxia, parkinsonism or chorea (discussed below).

In conclusion, FTD and ALS form a spectrum, with 'pure ALS' and 'pure FTD' at the extreme ends. The factors that modify the phenotypic expression and, thus, determine a patient's position on that continuum are unknown. The biological basis of such a disease continuum is likely to be complex, which is already evident from the observation that the 'FTD' component seems to be susceptible to different modifying factors than the 'ALS' component, as discussed below.

\section{ALS as a multisystem degeneration}

Although the symptoms and signs in an patient with ALS are predominantly motor in nature, it should be kept in mind that the lines defining neurodegenerative disorders are blurring. Some evidence for this phenomenon again comes from the study of C9orf $72^{(G G G G C C) e x p}$. This mutation gives rise to clinical characteristics well beyond ALS-FTD: it can also manifest as cerebellar abnormalities and autonomic dysfunction, leading to a diagnosis of multiple system atrophy, or as chorea and neuropsychiatric abnormalities, leading to a diagnosis of Huntington disease (HD). ${ }^{70,71}$

Surprisingly, some genes associated with ALS-FTD can also influence phenotypic traits that are not directly related to the function of the nervous system. Mutations in VCP, HNRNPA2B1 and HNRNPA 1 can induce ALS and/or FTLD combined with inclusion body myositis and bone abnormalities, such as those seen in Paget disease. ${ }^{72,73}$ These phenotypes again demonstrate the multisystemic character of ALS.

Evidence for the widespread nature of ALS-induced pathology has also come from the careful clinical observation of patients with unusually long survival in the 
setting of sustained ventilatory and nutritional support. In these patients, eye movement abnormalities, autonomic dysfunction and sensory involvement eventually become evident. ${ }^{74}$ In rare cases, such multisystem involvement is already apparent at disease onset, and sometimes the clinical presentation is so different from that of classic ALS that clinicians consider the patient to have a different disease. In the sections that follow, we discuss some of these presentations.

\section{ALS with extrapyramidal involvement}

Besides the endemic ALS-Parkinson dementia syndromes of Guam ${ }^{75}$ and the Kii peninsula, ${ }^{76}$ extrapyramidal involvement can sometimes be observed in patients with ALS. ALS associated with Parkinson disease (PD) is known as Brait-Fahn-Schwartz syndrome or ALS-PD complex, and is very rare. ${ }^{77}$ The parkinsonism in these patients is usually typical for $\mathrm{PD}$, including the response to levodopa ${ }^{78}$ Onset of motor neuron dysfunction usually follows a few years later, but simultaneous onset has been reported. ${ }^{77,79}$ Progression is similar to that of classic ALS. ${ }^{78}$ These rare patients are thought to have two separate diseases, although mutations in PARK7 (also known as DJ1) have been reported to cause a PD-ALS-FTD syndrome, providing a possible genetic link. ${ }^{80}$

ALS-parkinsonism is much more common than ALSPD complex. ALS-parkinsonism refers to the presence of extrapyramidal findings that are unresponsive to levodopa in patients with ALS. Mild parkinsonism is present in $5-15 \%$ of patients with ALS, and is typically characterized by postural instability and backward falls. ${ }^{78,81}$ In such patients, abnormalities in the nigrostriatal system can be seen on dopamine transporter imaging or at autopsy. ${ }^{78,81-83}$ In our experience, distinguishing the spastic, extrapyramidal and frontal motor findings in such patients is not trivial, which could explain why different studies have reported variable frequencies of ALS-parkinsonism.

Chorea and/or hemiballismus can occur late in the disease course in rare cases of ALS. ${ }^{84,85}$ As mentioned above, chorea is seen in some patients with a C9orf72 mutation. To date, about 15 cases of ALS-choreaconcomitant ALS and HD-have been reported. ${ }^{86}$ Most of these patients have late-onset ( $>50$ years) $\mathrm{HD}$, and developed ALS 5-10 years later. ${ }^{86}$ Neuropathological evidence for motor neuron involvement has been reported in a subset of patients with HD. ${ }^{87}$

\section{ALS and cerebellar ataxia}

Ataxia is a rare finding in ALS. As mentioned above, it is known to occur in patients with $C 90 r f 72^{\text {(GGGGCC) exp }}$. In addition, a few patients with spinocerebellar ataxia (SCA) have been reported to develop rapidly progressive ALS in the late stage of their disease (SCA-ALS) ${ }^{70,88}$ Interestingly, SCA type 2, which is caused by a CAG expansion in ATXN2, has genotypic and phenotypic overlap with ALS. Intermediate-length expansions in ATXN2 are a risk factor for ALS-but, strangely enough - not for ALS-FTD or FTD $^{89,90}$ — and ATXN2 mutations can present as ALS. ${ }^{91}$ The biological basis for the link between SCA and ALS is unknown.

\section{ALS with sensory involvement}

Subjective sensory symptoms are reported to occur in $50 \%$, and objective sensory signs in $10 \%$, of patients with ALS, but a true sensory neuropathy is rare in ALS. ${ }^{92}$ The SOD $1^{D 90 A}$ mutation gives rise to prominent sensory symptoms, with posterior column involvement evident at autopsy. ${ }^{14,93,94}$

\section{ALS with urinary and autonomic involvement} Apart from patients with a C9orf $72^{(G G G G C C) e x p}$ mutation, ${ }^{95}$ symptoms indicative of autonomic nervous system involvement are rare in ALS, although specific testing can indicate subclinical involvement of cardiovascular, gastrointestinal and sudomotor systems, and the salivary and lacrimal gland. ${ }^{96,97}$ Bladder problems are commonly believed not to be a feature of ALS, because Onuf's nucleus is typically spared or only minimally affected. ${ }^{3}$ Of note, urinary incontinence and retention certainly do occur in patients with ALS, but these problems are usually attributed to the use of muscle relaxants and anticholinergics, or to the patient's motor problems. However, it should be noted that patients with SOD1 Asp90Ala mutations often have urinary symptoms, ${ }^{14,94}$ and that urgency of micturition is a very frequent symptom of PLS, affecting $50-70 \%$ of patients. ${ }^{45,98}$

\section{ALS with ophthalmoplegia}

As mentioned above, ophthalmoplegia, resulting from widespread brainstem pathology, can be observed in very late stages of ALS, and is only seen in patients with unusually long survival; it very rarely occurs shortly after disease onset.

\section{ALS with deafness}

Deafness is not a feature of ALS or any of its genetic or phenotypic variants; however, it can be part of rare syndromes with ALS-like features, the most famous of which is Brown-Vialetto-Van Laere syndrome, a genetic disease caused by mutations in genes coding for riboflavin transporters. ${ }^{99}$ This syndrome is closely related to Fazio-Londe syndrome, in which auditory function is spared. ${ }^{3}$

\section{Summary}

In summary, the extrapyramidal, cerebellar, sensory or autonomic systems are rarely affected by ALS, and usually only with advanced disease. Similarly, urinary and ocular motor neuron involvement is only seen in patients with very long survival and advanced disease. Disruption of these systems usually has limited clinical importance, but it confirms the viewpoint that ALS is a multisystem neurological disease.

\section{From biology to therapy}

As described above, ALS has high phenotypic variability. Some of the phenotypic differences might be of a quantitative rather than a qualitative nature, as their detection depends on the method of observation. For example, if sophisticated testing is used, all patients with ALS might show evidence of frontal dysfunction, ${ }^{100}$ and transcranial 
magnetic stimulation of the cortex might uncover corticospinal abnormalities that would go unnoticed by a clinical examination. ${ }^{101}$

The molecular and cellular biology underlying the phenotypic variation remain elusive. Motor neurons seem strikingly vulnerable to ALS, those with a large diameter more so than small ones, ${ }^{102,103}$ whereas the oculomotor neurons and those in Onuf's nucleus are far more resistant. Why ALS starts in spinal neurons in some patients, but in bulbar neurons in others, remains puzzling. The large layer $\mathrm{V}$ neurons in the frontal and temporal cortex are generally less vulnerable than motor neurons, but in some patients this situation is reversed. Why do some patients with C9orf72(GGGGCC)exp develop ALS, whereas others develop FTD or ALS-FTD? Why do some patients with the SOD1 Gly93Cys mutations die after 2 years of disease, while others with the same mutation survive for more than 20 years? Why do some individuals carrying a pathogenic SOD1 or C9orf72 mutation never get ALS or FTD? Are they resistant to the disease, or is their age at onset beyond the normal human lifespan?

Several factors contributing to different aspects of this vulnerability have been identified, but the pathophysiological mechanisms remain poorly understood. The size of the motor neurons, the length of their axons, their metabolic rate and many other characteristics have been hypothesized as contributors, but never proven to have a role. Some evidence suggests that the low abundance of calcium buffering protein $\mathrm{s}^{104}$ and the presence of mitochondrial matrix metalloproteinase- $9^{105}$ underlie the difference in vulnerability between oculomotor and other motor neurons.Expression of the ephrin type-A receptor 4 protein (encoded by EPHA4), ${ }^{106}$ as well as excitability characteristics, ${ }^{107}$ have been suggested to contribute to the difference in vulnerability of large versus small motor neurons. Susceptibility of motor neurons to excitotoxicity could explain why spinal sensory neurons are less vulnerable to ALS than are motor neurons. ${ }^{108}$ Moreover, studies in SOD1 mutant mice suggest that the vulnerability of motor neurons is related to specific excitability-related pathways in these cells. ${ }^{107}$

Polymorphisms in the UNC13A gene and expression levels of EPHA4 have been suggested to contribute to the variability in disease severity. ${ }^{106,109}$ ATXN2 expansions are a risk factor for ALS but not for FTD, ${ }^{90}$ whereas the TMEM106B risk allele is a risk factor for FTD but not for ALS. ${ }^{110}$ Phenotypic variability across the ALS-FTD spectrum (and probably other spectra) is, thus, believed to be largely due to various genetic polymorphisms with different cytotoxic or cytoprotective effects according to neuronal cell type. Better knowledge of these factors, whether they be environmental or genetic in nature, is important, as they could be targets for intervention, even in patients in whom the cause of the disease is unknown.

\section{Conclusions}

Since its initial description by Charcot, ALS has been considered to be a homogenous neurodegenerative disease with selective involvement of both upper and lower motor neurons. Progress in genetics and in precise phenotyping of ALS has revealed shortcomings in the $19^{\text {th }}$ century semiological approach. Abundant evidence has redirected the view towards the concept of a heterogeneous multisystem neurodegenerative disease. Cognitive impairment is thought to be an intrinsic characteristic of this disease, as reflected in the ALS-FTD spectrum. Involvement of upper and lower motor neurons forms a similar spectrum. Variability in clinical presentation, onset, duration and hereditability is considerable. Clinically relevant involvement of extrapyramidal, cerebellar, autonomic and sensory systems is rare, but shows ALS to be a multisystem degeneration disorder.

Some of the clinical variability certainly depends on the method and timing of assessment, but the majority seems to reflect differences in vulnerability of neurons. Clinical, genetic and biological studies are underway to identify the factors that underlie this variability. It is unknown whether this variability also implies differential response to therapy. It remains to be seen whether all phenotypic presentations will respond to the same drug and, if so, to the same extent. Phenomics, genomics, proteomics, metabolomics and other unbiased approaches will need to be brought together. Such approaches will require large sets of very carefully and uniformly phenotyped patient populations. Hopefully, such studies will allow the development of strategies to change the phenotypic expression of ALS and find a cure for this dreadful disease.

\begin{tabular}{|c|}
\hline Review criteria \\
\hline 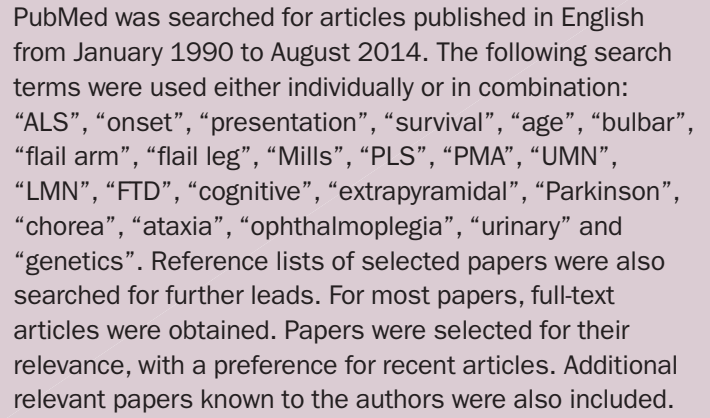 \\
\hline
\end{tabular}

1. Renton, A. E., Chiò, A. \& Traynor, B. J. State of play in amyotrophic lateral sclerosis genetics. Nat. Neurosci. 17, 17-23 (2014).

2. Robberecht, W. \& Philips, T. The changing scene of amyotrophic lateral sclerosis. Nat. Rev. Neurosci. 14, 248-264 (2013).

3. Amato, A. \& Russell, J. Neuromuscular Disorders 97-113 (McGraw-Hill, 2008).

4. Rowland, L. P. Amyotrophic lateral sclerosis. N. Engl. J. Med. 344, 1688-1700 (2001).
5. Ravits, J. M. \& La Spada, A. R. ALS motor phenotype heterogeneity, focality, and spread: deconstructing motor neuron degeneration. Neurology 73, 805-811 (2009).

6. Chiò, A., Calvo, A., Moglia, C., Mazzini, L. \& Mora, G. Phenotypic heterogeneity of amyotrophic lateral sclerosis: a population based study. J. Neurol. Neurosurg. Psychiatry 82, 740-746 (2011).
7. Duchenne De Boulogne, G. Paralysie musculaire progressive de la langue, du voile du palais et des levres [French]. Arch. Gen. Med. 2, 283-296 (1860).

8. Pinto, S., Pinto, A. \& De Carvalho, M. Do bulbaronset amyotrophic lateral sclerosis patients have an earlier respiratory involvement than spinal-onset amyotrophic lateral sclerosis patients? Eura Medicophys. 43, 505-509 (2007). 
9. Turner, M. R. et al. The diagnostic pathway and prognosis in bulbar-onset amyotrophic lateral sclerosis. J. Neurol. Sci. 294, 81-85 (2010).

10. Shoesmith, C. L., Findlater, K., Rowe, A. \& Strong, M. J. Prognosis of amyotrophic lateral sclerosis with respiratory onset. J. Neurol. Neurosurg. Psychiatry 78, 629-631 (2007).

11. Al-Chalabi, A. \& Hardiman, O. The epidemiology of ALS: a conspiracy of genes, environment and time. Nat. Rev. Neurol. 9, 617-628 (2013).

12. Sabatelli, M., Conte, A. \& Zollino, M. Clinical and genetic heterogeneity of amyotrophic lateral sclerosis. Clin. Genet. 83, 408-416 (2013).

13. Al-Chalabi, A. \& Lewis, C. M. Modelling the effects of penetrance and family size on rates of sporadic and familial disease. Hum. Hered. 71 281-288 (2011)

14. Andersen, P. M. \& Al-Chalabi, A. Clinical genetics of amyotrophic lateral sclerosis: what do we really know? Nat. Rev. Neurol. 7, 603-615 (2011).

15. Sabatelli, M. et al. Natural history of young-adult amyotrophic lateral sclerosis. Neurology 71 876-881 (2008)

16. Pupillo, E., Messina, P., Logroscino, G. \& Beghi, E. Long-term survival of amyotrophic lateral sclerosis: a population-based study. Ann. Neurol. 75, 287-297 (2014).

17. Belzil, V. V. et al. Novel FUS deletion in a patient with juvenile amyotrophic lateral sclerosis. Arch. Neurol. 69, 653-656 (2012).

18. Yamashita, S. et al. Sporadic juvenile amyotrophic lateral sclerosis caused by mutant FUS/TLS: possible association of mental retardation with this mutation. J. Neurol. 259, 1039-1044 (2012).

19. Bäumer, D. et al. Juvenile ALS with basophilic inclusions is a FUS proteinopathy with FUS mutations. Neurology 75, 611-618 (2010).

20. Dierick, I. et al. Relative contribution of mutations in genes for autosomal dominant distal hereditary motor neuropathies: a genotype-phenotype correlation study. Brain 131, 1217-1227 (2008).

21. Chen, Y.Z. et al. DNA/RNA helicase gene mutations in a form of juvenile amyotrophic lateral sclerosis (ALS4). Am. J. Hum. Genet. 74, 1128-1135 (2004).

22. Forbes, R. B., Colville, S. \& Swingler, R. J. The epidemiology of amyotrophic lateral sclerosis (ALS/MND) in people aged 80 or over. Age Ageing 33, 131-134 (2004).

23. Zoccolella, S. et al. Analysis of survival and prognostic factors in amyotrophic lateral sclerosis: a population based study. J. Neurol. Neurosurg. Psychiatry 79, 33-37 (2008).

24. Iwanaga, K. et al. Neuropathology of sporadic amyotrophic lateral sclerosis of long duration. J. Neurol. Sci. 146, 139-143 (1997).

25. Byrne, S. et al. Cognitive and clinical characteristics of patients with amyotrophic lateral sclerosis carrying a C9orf72 repeat expansion: a population-based cohort study. Lancet Neurol. 11, 232-240 (2012).

26. Chiò, A. et al. Extensive genetics of ALS : a population-based study in Italy. Neurology 79 , 1983-1989 (2012).

27. Van Rheenen, W. et al. Hexanucleotide repeat expansions in C9ORF72 in the spectrum of motor neuron diseases. Neurology 79, 878-882 (2012)

28. Van Blitterswijk, M. et al. Association between repeat sizes and clinical and pathological characteristics in carriers of C9ORF72 repeat expansions (Xpansize-72): a cross-sectional cohort study. Lancet Neurol. 12, 978-988 (2013)
29. Régal, L. et al. The G93C mutation in superoxide dismutase 1: clinicopathologic phenotype and prognosis. Arch. Neurol. 63, 262-267 (2006).

30. Penco, S. et al. Phenotypic heterogeneity in a SOD1 G93D Italian ALS family: an example of human model to study a complex disease. J. Mol. Neurosci. 44, 25-30 (2011).

31. Kim, W. et al. Anticipation and phenotypic heterogeneity in Korean familial amyotrophic lateral sclerosis with superoxide dismutase 1 gene mutation. J. Clin. Neurol. 3, 38-44 (2007).

32. Brooks, B. R. El Escorial World Federation of Neurology criteria for the diagnosis of amyotrophic lateral sclerosis. Subcommittee on Motor Neuron Diseases/Amyotrophic Lateral Sclerosis of the World Federation of Neurology Research Group on Neuromuscular Diseases and the El Escorial "Clinical limits of amyotrophic lateral sclerosis" workshop contributors. J. Neurol. Sci. 124, 96-107 (1994).

33. Rowland, L. P. Progressive muscular atrophy and other lower motor neuron syndromes of adults. Muscle Nerve 41, 161-165 (2010).

34. Cudkowicz, M., McKenna-Yasek, D., Chen, C., Hedley-Whyte, E. \& Brown, R. J. Limited corticospinal tract involvement in amyotrophic lateral sclerosis subjects with the A4V mutation in the copper/zinc superoxide dismutase gene. Ann. Neurol. 43, 703-710 (1998).

35. Cervenakova, L., Protas, I. \& Hirano, A. Progressive muscular atrophy variant of familial amyotrophic lateral sclerosis (PMA/ALS). J. Neurol. Sci. 177, 124-130 (2000)

36. Van der Graaff, M. M. et al. Upper and extramotoneuron involvement in early motoneuron disease: a diffusion tensor imaging study. Brain 134, 1211-1228 (2011).

37. Prudlo, J. et al. White matter pathology in ALS and lower motor neuron ALS variants: a diffusion tensor imaging study using tract-based spatial statistics. J. Neurol. 259, 1848-1859 (2012).

38. Ince, P. G. et al. Corticospinal tract degeneration in the progressive muscular atrophy variant of ALS. Neurology 60, 1252-1258 (2003).

39. Gamez, J., Cervera, C. \& Codina, A. Flail arm syndrome or Vulpian-Bernhart's form of amyotrophic lateral sclerosis. J. Neurol. Neurosurg. Psychiatry 67, 258 (1999).

40. Hu, M., Ellis, C., Al-Chalabi, A., Leigh, P. \& Shaw, C. Flail arm syndrome: a distinctive variant of amyotrophic lateral sclerosis. J. Neurol Neurosurg. Psychiatry 65, 950-951 (1998).

41. Wijesekera, L. C. et al. Natural history and clinical features of the flail arm and flail leg ALS variants. Neurology 72, 1087-1094 (2009).

42. Kobayashi, Z. et al. Pseudopolyneuritic form of ALS revisited: clinical and pathological heterogeneity. Neuropathology 30, 372-380 (2010).

43. Gourie-Devi, M., Nalini, A. \& Sandhya, S. Early or late appearance of "dropped head syndrome" in amyotrophic lateral sclerosis. J. Neurol. Neurosurg. Psychiatry 74, 683-687 (2003).

44. Gordon, P. H. et al. The natural history of primary lateral sclerosis. Neurology 66, 647-653 (2006).

45. Le Forestier, N. et al. Does primary lateral sclerosis exist? A study of 20 patients and a review of the literature. Brain 124, 1989-1999 (2001).

46. D'amico, E., Pasmantier, M. \& Lee, Y.-W. Clinical evolution of pure upper motor neuron disease/ dysfunction (PUMND). Muscle Nerve 47, 28-32 (2013).

47. Gordon, P., Cheng, B. \& Katz, I. Clinical features that distinguish PLS, upper motor neurondominant ALS, and typical ALS. Neurology 72 , 1947-1953 (2009).
48. Rajabally, Y. A., Hbahbih, M. \& Abbott, R. J. Hemiplegic ALS: Mills syndrome. Neurology 64 , 1984-1985 (2005).

49. Gastaut, J.-L. \& Bartolomei, F. Mills' syndrome: ascending (or descending) progressive hemiplegia: a hemiplegic form of primary latera sclerosis? J. Neurol. Neurosurg. Psychiatry 57 1280-1281 (1994).

50. Kim, W.-K. et al. Study of 962 patients indicates progressive muscular atrophy is a form of ALS. Neurology 73, 1686-1692 (2009).

51. Van den Berg-Vos, R. M. et al. A long-term prospective study of the natural course of sporadic adult-onset lower motor neuron syndromes. Arch. Neurol. 66, 751-757 (2009).

52. Strong, M. J. \& Yang, W. The frontotemporal syndromes of ALS. Clinicopathological correlates. J. Mol. Neurosci. 45, 648-655 (2011).

53. Phukan, J., Pender, N. P. \& Hardiman, O. Cognitive impairment in amyotrophic lateral sclerosis. Lancet Neurol. 6, 994-1003 (2007).

54. Lillo, P. \& Hodges, J. R. Frontotemporal dementia and motor neurone disease: overlapping clinicpathological disorders. J. Clin. Neurosci. 16, 1131-1135 (2009).

55. Mioshi, E. et al. Neuropsychiatric changes precede classic motor symptoms in ALS and do not affect survival. Neurology 82, 149-155 (2014)

56. Rippon, G. A. et al. An observational study of cognitive impairment in amyotrophic lateral sclerosis. Arch. Neurol. 63, 345-352 (2006).

57. Ferrari, R., Kapogiannis, D., Huey, E. D. \& Momeni, P. FTD and ALS: a tale of two diseases. Curr. Alzheimer Res. 8, 273-294 (2011).

58. Olney, R. K. et al. The effects of executive and behavioral dysfunction on the course of ALS. Neurology 65, 1774-1777 (2005).

59. Achi, E. Y. \& Rudnicki, S. A. ALS and frontotemporal dysfunction: a review. Neurol. Res. Int. 2012, 806306 (2012).

60. Neumann, M. et al. Ubiquitinated TDP-43 in frontotemporal lobar degeneration and amyotrophic lateral sclerosis. Science $\mathbf{3 1 4}$, 130-133 (2006).

61. DeJesus-Hernandez, M. et al. Expanded GGGGCC hexanucleotide repeat in noncoding region of C9ORF72 causes chromosome 9p-linked FTD and ALS. Neuron 72, 245-256 (2011)

62. Renton, A. E. et al. A hexanucleotide repeat expansion in C9ORF72 is the cause of chromosome 9p21-linked ALS-FTD. Neuron 72, 257-268 (2011)

63. Gijselinck, I. et al. A C9orf72 promoter repeat expansion in a Flanders-Belgian cohort with disorders of the frontotemporal lobar degeneration-amyotrophic lateral sclerosis spectrum: a gene identification study. Lancet Neurol. 11, 54-65 (2012).

64. Cooper-Knock, J., Shaw, P. J. \& Kirby, J. The widening spectrum of C9ORF72-related disease genotype/phenotype correlations and potential modifiers of clinical phenotype. Acta Neuropathol. 127, 333-345 (2014).

65. Snowden, J. S. et al. Distinct clinical and pathological characteristics of frontotemporal dementia associated with C9ORF72 mutations. Brain 135, 693-708 (2012).

66. Cudkowicz, M. et al. Epidemiology of mutations in superoxide dismutase in amyotrophic lateral sclerosis. Ann. Neurol. 41, 210-221 (1997).

67. Millecamps, S. et al. Phenotype difference between ALS patients with expanded repeats in C9ORF72 and patients with mutations in other ALS-related genes. J. Med. Genet. 49, 258-263 (2012). 
68. Hodges, J. Familial frontotemporal dementia and amyotrophic lateral sclerosis associated with the C9ORF72 hexanucleotide repeat. Brain 135 , 652-655 (2012).

69. Bieniek, K. F. et al. Expanded C9ORF72 hexanucleotide repeat in depressive pseudodementia. JAMA Neurol. 71, 775-781 (2014).

70. Lindquist, S. G. et al. Corticobasal and ataxia syndromes widen the spectrum of C9ORF72 hexanucleotide expansion disease. Clin. Genet. 83, 279-283 (2013).

71. Hensman Moss, D. J. et al. C9orf72 expansions are the most common genetic cause of Huntington disease phenocopies. Neurology 82, 292-299 (2014).

72. Watts, G. D. et al. Inclusion body myopathy associated with Paget disease of bone and frontotemporal dementia is caused by mutant valosin-containing protein. Nat. Genet. 36, 377-381 (2004).

73. Kim, D. H. J. et al. Mutations in prion-like domains in hnRNPA2B1 and hnRNPA1 cause multisystem proteinopathy and ALS. Nature 495 , 467-473 (2013).

74. Atsuta, N. et al. Age at onset influences on wideranged clinical features of sporadic amyotrophic lateral sclerosis. J. Neurol. Sci. 276, 163-169 (2009).

75. Steele, J. C. \& McGeer, P. L. The ALS/PDC syndrome of Guam and the cycad hypothesis. Neurology 70, 1984-1990 (2008).

76. Kaji, R., Izumi, Y., Adachi, Y. \& Kuzuhara, S. ALSparkinsonism-dementia complex of Kii and other related diseases in Japan. Parkinsonism Relat. Disord. 18 (Suppl. 1), S190-S191 (2012).

77. Gilbert, R. M. W., Fahn, S., Mitsumoto, H. \& Rowland, L. P. Parkinsonism and motor neuron diseases: twenty-seven patients with diverse overlap syndromes. Mov. Disord. 25, 1868-1875 (2010).

78. Manno, C., Lipari, A., Bono, V., Taiello, A. C. $\&$ La Bella, V. Sporadic Parkinson disease and amyotrophic lateral sclerosis complex (BraitFahn-Schwartz disease). J. Neurol. Sci. 326, 104-106 (2013)

79. Pinkhardt, E. H., Sperfeld, A.-D., Gdynia, H.-J., Ludolph, A. C. \& Kassubek, J. The combination of dopa-responsive parkinsonian syndrome and motor neuron disease. Neurodegener. Dis. 6 , 95-101 (2009).

80. Annesi, G. et al. DJ-1 mutations and parkinsonism-dementia-amyotrophic lateral sclerosis complex. Ann. Neurol. 58, 803-807 (2005).

81. Desai, J. \& Swash, M. Extrapyramidal involvement in amyotrophic lateral sclerosis: backward falls and retropulsion. J. Neurol. Neurosurg. Psychiatry 67, 214-216 (1999).

82. Park, H. K. et al. Nigrostriatal dysfunction in patients with amyotrophic lateral sclerosis and parkinsonism. J. Neurol. Sci. 301, 12-13 (2011).

83. Frolov, A. et al. C9ORF72 expansions, parkinsonism, and parkinson disease. Neurology 81, 808-811 (2013).
84. Pradat, P.-F. Association of chorea and motor neuron disease. Mov. Disord. 17, 402-423 (2002).

85. Gamez, J. et al. Chorea-ballism associated with familial amyotrophic lateral sclerosis. A clinical, genetic, and neuropathological study. Mov. Disord. 23, 434-438 (2008).

86. Tada, M. et al. Coexistence of Huntington's disease and amyotrophic lateral sclerosis: a clinicopathologic study. Acta Neuropathol. 124, 749-760 (2012).

87. Sadeghian, H., O'Suilleabhain, P. E., Battiste, J. Elliott, J. L. \& Trivedi, J. R. Huntington chorea presenting with motor neuron disease. Arch. Neurol. 68, 650-652 (2011).

88. Nanetti, L. et al. Rare association of motor neuron disease and spinocerebellar ataxia type 2 (SCA2): a new case and review of the literature. J. Neurol. 256, 1926-1928 (2009).

89. Elden, A. C. et al. Ataxin-2 intermediate-length polyglutamine expansions are associated with increased risk for ALS. Nature 466, 1069-1075 (2011).

90. Ross, O. A. et al. Ataxin-2 repeat-length variation and neurodegeneration. Hum. Mol. Genet. 20, 3207-3212 (2011)

91. Van Damme, P. et al. Expanded ATXN2 CAG repeat size in ALS identifies genetic overlap between ALS and SCA2. Neurology 76 2066-2072 (2011).

92. Isaacs, J. D. et al. Amyotrophic lateral sclerosis with sensory neuropathy: part of a multisystem disorder? J. Neurol. Neurosurg. Psychiatry 78, 750-753 (2007).

93. Shibata, N. et al. Intense superoxide dismutase-1 immunoreactivity in intracytoplasmic hyaline inclusions of familial amyotrophic lateral sclerosis with posterior column involvement. J. Neuropathol. Exp. Neurol. 55, 481-490 (1996).

94. Andersen, P. M. et al. Amyotrophic lateral sclerosis associated with homozygosity for an Asp90Ala mutation in CuZn-superoxide dismutase. Nat. Genet. 55, 61-66 (1995).

95. Lee, H. L. \& Lee, J. K. Amyotrophic lateral sclerosis with an acute hypertensive crises. Ann. Rehabil. Med. 36, 418-422 (2012).

96. Baltadzhieva, R., Gurevich, T. \& Korczyn, A. D. Autonomic impairment in amyotrophic lateral sclerosis. Curr. Opin. Neurol. 18, 487-493 (2005).

97. Merico, A. \& Cavinato, M. Autonomic dysfunction in the early stage of ALS with bulbar involvement. Amyotroph. Lateral Scler. 12 , 363-367 (2011).

98. Zhai, P., Pagan, F., Statland, J., Butman, J. A \& Floeter, M. K. Primary lateral sclerosis: a heterogeneous disorder composed of different subtypes? Neurology 60, 1258-1265 (2003).

99. Green, P. et al. Brown-Vialetto-Van Laere syndrome, a ponto-bulbar palsy with deafness, is caused by mutations in C200RF54. Am. J. Hum. Genet. 86, 485-489 (2010).

100. Abrahams, S. et al. Frontal lobe dysfunction in amyotrophic lateral sclerosis. A PET study. Brain 119, 2105-2120 (1996).
101. Chervyakov, A. V. et al. Navigated transcranial magnetic stimulation in amyotrophic lateral sclerosis. Muscle Nerve 9, 408-419 (2014).

102. Pun, S., Santos, A. F., Saxena, S., Xu, L. \& Caroni, P. Selective vulnerability and pruning of phasic motoneuron axons in motoneuron disease alleviated by CNTF. Nat. Neurosci. 9 , 408-419 (2006)

103. Frey, D. et al. Early and selective loss of neuromuscular synapse subtypes with low sprouting competence in motoneuron diseases. J. Neurosci. 20, 2534-2542 (2000).

104. Vanselow, B. K. \& Keller, B. U. Calcium dynamics and buffering in oculomotor neurones from mouse that are particularly resistant during amyotrophic lateral sclerosis (ALS)-related motoneurone disease. J. Physiol. 525, 433-445 (2000)

105. Kaplan, A. et al. Neuronal matrix metalloproteinase- 9 is a determinant of selective neurodegeneration. Neuron $\mathbf{8 1}$, 333-348 (2014).

106. Van Hoecke, A. et al. EPHA4 is a disease modifier of amyotrophic lateral sclerosis in animal models and in humans. Nat. Med. 18, 1418-1422 (2012).

107. Saxena, S. et al. Neuroprotection through excitability and mTOR required in ALS motoneurons to delay disease and extend survival. Neuron 80, 80-96 (2013).

108. Van Den Bosch, L., Van Damme, P., Bogaert, E. $\&$ Robberecht, W. The role of excitotoxicity in the pathogenesis of amyotrophic lateral sclerosis. Biochim. Biophys. Acta 1762, 1068-1082 (2006).

109. Diekstra, F. P. et al. UNC13A is a modifier of survival in amyotrophic lateral sclerosis. Neurobiol. Aging 33, 630.e3-630.e8 (2012).

110. Vass, R., Hu, W. T. \& Grossman, M. Risk genotypes at TMEM106B are associated with cognitive impairment in amyotrophic lateral sclerosis. Acta Neuropathol. 121, 373-380 (2011).

\section{Acknowledgements}

B.S. is funded by the Fund for Scientific Research Flanders FWO (11Y9515N). W.R. is funded by the European Research Council (under the European Commission Seventh Framework Programme [FP7/2007-2013 under grant agreements \#340429 and \#259867), the Fund for Scientific Research Flanders (FWO; G.0983.14N and G.0996.14N), the University of Leuven (GOA/11/014 and Fund Hart voor ALS), the Interuniversity Attraction Poles Programme (P7/16), the ALS association (039CYK), the Packard Center for ALS research, the Thierry Latran Foundation and the ALS Therapy Alliance. The authors are grateful to P. N. Leigh for reading and commenting on the manuscript.

\section{Author contributions}

B.S. and W.R. researched data for the article. Both authors made substantial contributions to discussions of the content, writing the article, and reviewing and/or editing of the manuscript before submission. 